\title{
Thermoregulation During Intermittent Exercise in Athletes With a Spinal-Cord Injury
}

\author{
Katy E. Griggs, Christof A. Leicht, Michael J. Price, and Victoria L. Goosey-Tolfrey
}

\begin{abstract}
Purpose: Individuals with a spinal-cord injury have impaired thermoregulatory control due to a loss of sudomotor and vasomotor effectors below the lesion level. Thus, individuals with high-level lesions (tetraplegia) possess greater thermoregulatory impairment than individuals with lower-level lesions (paraplegia). Previous research has not reflected the intermittent nature and modality of wheelchair court sports or replicated typical environmental temperatures. Hence, the purpose of this study was to investigate the thermoregulatory responses of athletes with tetraplegia and paraplegia during an intermittent-sprint protocol (ISP) and recovery in cool conditions. Methods: Sixteen wheelchair athletes, 8 with tetraplegia (TP, body mass $65.2 \pm 4.4 \mathrm{~kg}$ ) and 8 with paraplegia (body mass $68.1 \pm 12.3 \mathrm{~kg}$ ), completed a 60 -min ISP in $20.6^{\circ} \mathrm{C} \pm 0.1^{\circ} \mathrm{C}, 39.6 \% \pm 0.8 \%$ relative humidity on a wheelchair ergometer, followed by 15 min of passive recovery. Core temperature $\left(T_{\text {core }}\right)$ and mean $\left(T_{\mathrm{sk}}\right)$ and individual skin temperatures were measured throughout. Results: Similar external work $(P=.70, \mathrm{ES}=0.20)$ yet a greater $T_{\text {core }}(P<.05, \mathrm{ES}=$ $2.27)$ and $T_{\mathrm{sk}}(P<.05, \mathrm{ES}=1.50)$ response was demonstrated by TP during the ISP. Conclusions: Despite similar external work, a marked increase in $T_{\text {core }}$ in TP during exercise and recovery signifies that thermoregulatory differences between the groups were predominantly due to differences in heat loss. Further increases in thermal strain were not prevented by the active and passive recovery between maximal-effort bouts of the ISP, as $T_{\text {core }}$ continually increased throughout the protocol in TP.
\end{abstract}

Keywords: thermoregulatory, intermittent-sprint exercise, wheelchair sport, tetraplegia, paraplegia.

Individuals with a spinal-cord injury (SCI) have reduced afferent information to the thermoregulatory center ${ }^{1,2}$ and a loss of both sweating capacity and vasomotor control below the level of the spinal lesion. ${ }^{1,3,4}$ As blood-flow redistribution and sweating are 2 major thermoregulatory effectors, this suggests that individuals with an SCI have compromised thermoregulation and are at a greater risk of heat illness than able-bodied individuals. ${ }^{5}$

The magnitude of the thermoregulatory impairment in those with an SCI is proportional to the level of the lesion. Exercising for 60 to 90 minutes at $60 \% \mathrm{VO}_{2 \text { peak }}$ in $15^{\circ} \mathrm{C}$ to $25^{\circ} \mathrm{C}$, trained individuals with a thoracic, lumbar, or sacral SCI (paraplegia) may experience an increase in core temperature $\left(T_{\text {core }}\right)$ similar to their able-bodied counterparts $\left(\sim 1^{\circ} \mathrm{C}\right) .{ }^{5}$ In hot conditions $\left(30-40^{\circ} \mathrm{C}\right)$ at the same exercise intensity, greater increases in $T_{\text {core }}$ are demonstrated than in the able-bodied, with even greater increases apparent in untrained individuals. ${ }^{6,7}$ Individuals with a cervical SCI (tetraplegia [TP]) possess a smaller area of sensate skin, a lesser amount of afferent input regarding their thermal state, and a reduced efferent response compared with individuals with paraplegia (PA). ${ }^{4,8}$ Less is known regarding the thermoregulatory responses of athletes with TP during exercise, yet it is thought they may experience a disproportionate increase in $T_{\text {core }}$ and heat storage, due to the presence of little or no sweating response, leading to a greater degree of thermal strain. ${ }^{9}$ Price and Campbell ${ }^{9}$ demonstrated that an athlete with TP arm cranking at $60 \% \mathrm{VO}_{2 \text { peak }}$ for 60 minutes in $\sim 21.5^{\circ} \mathrm{C}$ experienced

Griggs, Leicht, and Goosey-Tolfrey are with the Peter Harrison Centre for Disability Sport, Loughborough University, Loughborough, UK. Price is with the Dept of Biomolecular and Sports Science, Coventry University, Coventry, UK. Address author correspondence to Victoria Goosey-Tolfrey at v.l.tolfrey@lboro.ac.uk. a continuous increase in $T_{\text {core }}$, in contrast to a plateau experienced by able-bodied athletes and those with PA. While the athlete with TP did not experience high thermal strain in these conditions, the continuous rise in $T_{\text {core }}$ shows that thermal balance was not achieved.

Previous research has predominantly used arm-cranking protocols ${ }^{6,10}$ to examine the thermoregulation of athletes with SCI, not their habitual mode of wheelchair exercise. However, thermoregulatory differences exist between different modalities, with lower physiological and thermal strain elicited during wheelchair propulsion, due to intermittent application of force to the flywheel, than with continuous force application during arm cranking. ${ }^{11}$ Moreover, previous studies have not matched the ambient conditions to indoor playing environments or the intermittent nature of wheelchair court sports such as wheelchair basketball and rugby. Therefore, the purpose of this study was to compare the thermoregulatory responses of athletes with PA and TP during intermittent-sprint wheelchair exercise and recovery in cool conditions.

\section{Methods}

\section{Participants}

Eight wheelchair rugby players with TP (7 men, 1 woman, 1 incomplete lesion) ${ }^{12}$ and 8 wheelchair basketball players with PA (7 men, 1 woman, 3 incomplete lesions $)^{12}$ (Table 1), gave their written informed consent to participate in this experimental research study. The study was approved by the university research ethics committee and was conducted in accordance with the Declaration of Helsinki.

\section{Preliminary Tests}

On arrival at the laboratory, skinfold measurements were taken from the biceps, triceps, subscapular, suprailiac, and abdomen, followed 
Table 1 Physiological and Participant Characteristics of Athletes With Tetraplegia and Paraplegia, Mean \pm SD

\begin{tabular}{lcc}
\hline & Tetraplegia & Paraplegia \\
\hline Age (y) & $27.4 \pm 4.2$ & $27.8 \pm 6.2$ \\
Body mass (kg) & $65.2 \pm 4.4$ & $67.7 \pm 13.1$ \\
Sum of skinfolds (mm) & $65.4 \pm 28.2$ & $78.2 \pm 38.2$ \\
$\mathrm{VO}_{2 \text { peak }}(\mathrm{L} / \mathrm{min})$ & $1.55 \pm 0.37$ & $1.92 \pm 0.47$ \\
Lesion level (range) & $\mathrm{C} 4 / 5-\mathrm{C} 6 / 7$ & $\mathrm{~T} 4-\mathrm{S} 1$ \\
Time since injury (y) & $8.0 \pm 4.6$ & $11.4 \pm 7.7$ \\
Training (h/wk) & $15.0 \pm 4.2$ & $11.0 \pm 6.4$ \\
\hline
\end{tabular}

by a continuous incremental test on a treadmill to determine peak oxygen uptake $\left(\mathrm{VO}_{2 \text { peak }}\right)$. For the $\mathrm{VO}_{2 \text { peak }}$ test, participants were set up in their own sports wheelchair and mounted on the treadmill; workload was increased by 0.2 or $0.3 \mathrm{~m} / \mathrm{s}$ every 3 minutes (dependent on the individual's classification) until the participant could no longer maintain the speed of the treadmill.

\section{Experimental Conditions}

Participants ingested a telemetry pill (HQ Inc, Palmetto, Florida) for the measurement of core temperature $\left(T_{\text {core }}\right) \sim 8$ hours before the start of the test to avoid the influence of ingested food or fluid on the temperature reading. Two hours after the preliminary test, participants were weighed (Marsden Weighing Group Ltd, Henleyon-Thames, UK) with no clothing covering their upper body. During the intermittent-sprint protocol (ISP) participants wore their usual training attire of lightweight tracksuit trousers and either a short- or long-sleeved top. Seven thermistors (Grant Instruments, Cambridge, UK) were attached to the skin using strips of water-permeable surgical tape (3M Transpore, Loughborough) placed on the right side of the body on the forehead, forearm, biceps, upper back, chest, thigh, and calf for measurement of skin temperature (Grant Squirrel logger, Series 2010, Grant Instruments, Cambridge, UK). Mean skin temperature $\left(T_{\mathrm{sk}}\right)$ was estimated in accordance with the formula by Ramanathan. ${ }^{13}$ Heat storage was calculated using the following formula ${ }^{14}$ :

$$
\text { Heat storage }=\left(0.8 \Delta T_{\text {core }}+0.2 \Delta T_{\mathrm{sk}}\right) \times c_{\mathrm{b}}
$$

where $c_{\mathrm{b}}$ is the specific heat capacity of the body tissue $\left(3.49 \mathrm{~J} \cdot \mathrm{g}^{-1}\right.$ $\cdot{ }^{\circ} \mathrm{C}^{-1}$ ) and $\Delta T_{\text {core }}$ and $\Delta T_{\text {sk }}$ represent changes in $T_{\text {core }}$ and $T_{\text {sk }}$ from rest to the end of each exercise block and recovery. An estimate of external work was calculated by total distance covered (m) during the ISP multiplied by total resistance $(\mathrm{N})$ of the ergometerwheelchair system.

After instrumentation and transfer to their own sports wheelchair, participants rested for 10 minutes before completing a self-selected warm-up on a single-cylinder wheelchair ergometer (WERG, Bromakin, Loughborough, UK). ${ }^{15}$ During the warm-up, they performed a deceleration test for power and resistance to be calculated. ${ }^{16}$

The ISP was conducted in an environmental chamber at $20.6^{\circ} \mathrm{C}$ $\pm 0.1^{\circ} \mathrm{C}$ and $39.6 \% \pm 0.8 \%$ relative humidity, chosen to replicate a sports-hall environment. All participants completed the test at a similar time in the afternoon to negate circadian variation, and they refrained from caffeine and alcohol 24 hours before the test. The ISP simulated an on-court session and is reported elsewhere. ${ }^{17}$ Briefly, it consisted of 4 exercise blocks separated by 4.5 minutes of passive recovery (Figure 1). Each block comprised 6 bouts of 30 seconds, where athletes performed alternately 3 pushes forward and backward for the first 15 seconds followed by a 15 -second sprint at maximum effort. Bouts were followed by 90 seconds of active recovery at low intensity. At the end of block 4, participants rested for 15 minutes before all thermistors were removed and they were reweighed. The whole session lasted 55.5 minutes, with maximum-intensity activity accounting for 12 minutes, including a total of 24 sprints. Verbal encouragement was given throughout the test.

Heart rate (HR) was recorded at 5-second intervals during the ISP (Polar PE 4000, Kempele Finland). Whole-body rating of perceived exertion ${ }^{18}$ and thermal sensation ${ }^{19}$ were recorded at the end of each exercise block. Before the start of the ISP and during recovery, thermal sensation was also recorded. The thermal-sensation scale comprised categories ranging from 0 (unbearably cold) to 8 (unbearably hot). After the warm-up and on completion of exercise, capillary blood samples were taken from the earlobe and analyzed for hematocrit (Haemtospin 1300, Hawksley, Lancing, UK) and hemoglobin (B-Hemoglobin, Hemocue Ltd, Dronfield, UK) to determine plasma volume. ${ }^{20}$ Capillary blood samples were taken at the end of each block for analysis of blood lactate concentration (YSI Sport, YSI Inc, OH, USA). Participants were allowed to drink ad libitum during the passive recovery between blocks.

\section{Statistical Analysis}

All data were checked for normality, using the Shapiro-Wilk test. Delta core and skin temperatures were calculated. Independent $t$ tests were used to analyze any between-groups differences in participant characteristics, total distance, total resistance, external work, fluid balance, and start and end $T_{\text {core }}, T_{\text {sk }}$, and heat storage. Sprint speed and power output across the 24 sprints and physiological and thermoregulatory responses were analyzed using a 2 -way (group $\times$ time) analysis of variance (ANOVA). Where significance was obtained, post hoc pairwise comparisons with a Bonferroni correction were conducted. For individual skin temperatures and heat storage during recovery, data from 7 athletes with TP were used, as data from the last 3 minutes of recovery were missing for 1 participant. For all comparisons where the assumption of sphericity was violated, a Greenhouse-Geisser correction was applied. Effect sizes (ES) were estimated by Cohen $d$, where 0.2 represented a small effect size, 0.5 a medium effect size, and 0.8 a large effect size. ${ }^{21}$ All data were analyzed using SPSS version 19.0, and significance was accepted at the $P \leq .05$ level.

\section{Results}

\section{Participant Characteristics}

There were no differences between TP and PA for the physiological and participant characteristics $(P>.05$, Table 1$)$, yet large effect sizes were apparent for $\mathrm{VO}_{2 \text { peak }}(\mathrm{ES}=0.89)$ and training hours per week $(\mathrm{ES}=0.73)$.

\section{Sprint Performance}

There were no differences between groups or across the 24 sprints for either sprint speed or peak power output (all $P>.05$, Table 2). Total resistance of the ergometer-wheelchair system was greater in $\mathrm{TP}(P=.01, \mathrm{ES}=1.64)$, while total distance covered during the ISP was greater for PA $(P<.001, \mathrm{ES}=1.92)$. External work was not statistically different between groups $(P=.70, \mathrm{ES}=0.20)$. 


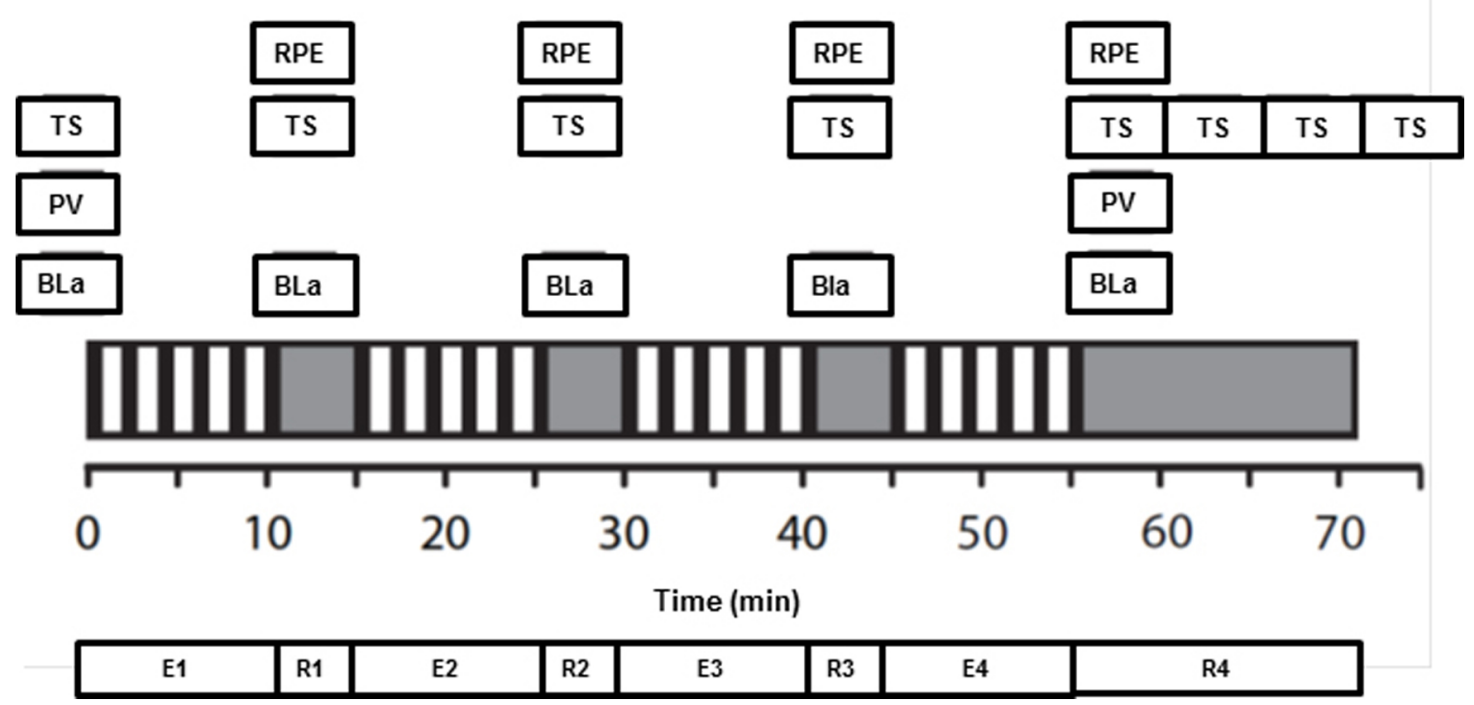

Figure 1 - Schematic of the intermittent-sprint protocol (ISP), including all measures taken throughout the 4 exercise blocks and recovery. The black blocks depict both the 15 seconds of alternate forward and backward pushing and the 15 -second sprints. The white blocks depict the 90 seconds of active recovery. The gray blocks show the 4.5 minutes of passive recovery between exercise blocks and the 15 minutes of recovery after the ISP. The corresponding exercise blocks and recovery periods are numbered below the time axis, and Figures 2 and 3 refer to these labels. Warm-up is not included in the figure. Abbreviations: RPE, rating of perceived exertion; TS, thermal sensation; PV, measures to determine plasma volume (hemoglobin and hematocrit); BLa, blood lactate; E, exercise block; R, passive recovery.

Table 2 Sprint Performance for Athletes With Tetraplegia and Paraplegia, Mean \pm SD

\begin{tabular}{lcc}
\hline & Tetraplegia & Paraplegia \\
\hline Sprint speed $(\mathrm{m} / \mathrm{s})^{\mathrm{a}}$ & $3.14 \pm 0.59$ & $3.51 \pm 0.44$ \\
Peak power output $(\mathrm{W})^{\mathrm{a}}$ & $67 \pm 14$ & $59 \pm 14$ \\
Total resistance $(\mathrm{N})$ & $21 \pm 3^{*}$ & $17 \pm 3$ \\
Total distance $(\mathrm{m})$ & $2316 \pm 258^{*}$ & $3042 \pm 468$ \\
External Work $(\mathrm{kJ})$ & $49 \pm 5$ & $51 \pm 9$ \\
\hline
\end{tabular}

a Sprint speed and power output across the 24 sprints.

*Significantly different from paraplegia $(P<.05)$

\section{Physiological Responses}

Mean and peak HR for each block of the ISP were greater for PA than TP $(P<.05$, Table 3$)$. Mean HR for both groups increased from block 1 to 2 and then remained stable throughout exercise. For both groups peak HR was similar over time $(P=.43)$. Throughout exercise, blood lactate was similar over time $(P=.09)$ but different between groups $(8.08 \pm 3.04$ and $8.73 \pm 2.17 \mathrm{mmol} / \mathrm{L}$ for TP and $\mathrm{PA}$, respectively, $P=.02, \mathrm{ES}=0.25)$.

\section{Core Temperature}

$T_{\text {core }}$ was similar between groups at the start of exercise $\left(37.0^{\circ} \mathrm{C} \pm\right.$ $0.6^{\circ} \mathrm{C}$ and $37.1^{\circ} \mathrm{C} \pm 0.3^{\circ} \mathrm{C}$ for TP and PA, respectively, $P=.75, \mathrm{ES}=$ $0.16)$. At the end of exercise TP demonstrated a greater $T_{\text {core }}$ than PA
Table 3 Mean and Peak Heart Rate and Blood Lactate During the Intermittent-Sprint Protocol for Athletes With Tetraplegia and Paraplegia, Mean \pm SD

\begin{tabular}{lcc}
\hline & Tetraplegia & Paraplegia \\
\hline Mean heart rate (beats/min) & $107 \pm 6^{*}$ & $132 \pm 15$ \\
Peak heart rate (beats/min) & $133 \pm 6^{*}$ & $161 \pm 8$ \\
Blood lactate (mmol/L) & $8.08 \pm 3.04^{*}$ & $8.73 \pm 2.16$ \\
\hline
\end{tabular}

*Significantly different from paraplegia $(P<.05)$.

$\left(38.2^{\circ} \mathrm{C} \pm 0.5^{\circ} \mathrm{C}\right.$ and $37.6^{\circ} \mathrm{C} \pm 0.4^{\circ} \mathrm{C}$ for TP and PA, respectively, $P=$ $.02, \mathrm{ES}=1.32$ ). During both exercise and recovery, TP experienced a greater increase in $T_{\text {core }}$ from resting values than PA (both $P<.0001$, $\mathrm{ES}=0.75$ and $\mathrm{ES}=2.27$ for exercise and recovery, respectively, Figure 2). At the end of recovery, $T_{\text {core }}$ for TP remained elevated from rest by $1.1^{\circ} \mathrm{C}$ compared with $0.2^{\circ} \mathrm{C}$ for PA $\left(38.1^{\circ} \mathrm{C} \pm 0.5^{\circ} \mathrm{C}\right.$ and $37.3^{\circ} \mathrm{C}$ $\pm 0.3^{\circ} \mathrm{C}$ for TP and PA, respectively, $P<.001$, ES $=1.84$ ).

\section{Skin Temperature}

$T_{\text {sk }}$ was similar between groups at the start $\left(29.5^{\circ} \mathrm{C} \pm 1.6^{\circ} \mathrm{C}\right.$ and $30.6^{\circ} \mathrm{C} \pm 0.6^{\circ} \mathrm{C}$ for TP and PA, respectively, $P=.09, \mathrm{ES}=0.91$ ) and end of exercise $\left(30.2^{\circ} \mathrm{C} \pm 1.5^{\circ} \mathrm{C}\right.$ and $30.0^{\circ} \mathrm{C} \pm 1.6^{\circ} \mathrm{C}$ for TP and PA, respectively, $P=.75, \mathrm{ES}=0.16)$ and end of recovery $\left(30.0^{\circ} \mathrm{C} \pm 1.4^{\circ} \mathrm{C}\right.$ and $29.7^{\circ} \mathrm{C} \pm 1.8^{\circ} \mathrm{C}$ for TP and PA, respectively, $P=.76, \mathrm{ES}=0.16$ ). During exercise and recovery the change in $T_{\mathrm{sk}}$ from resting values was different between TP and PA $(P<.001, \mathrm{ES}=1.50, P=.02$, ES 


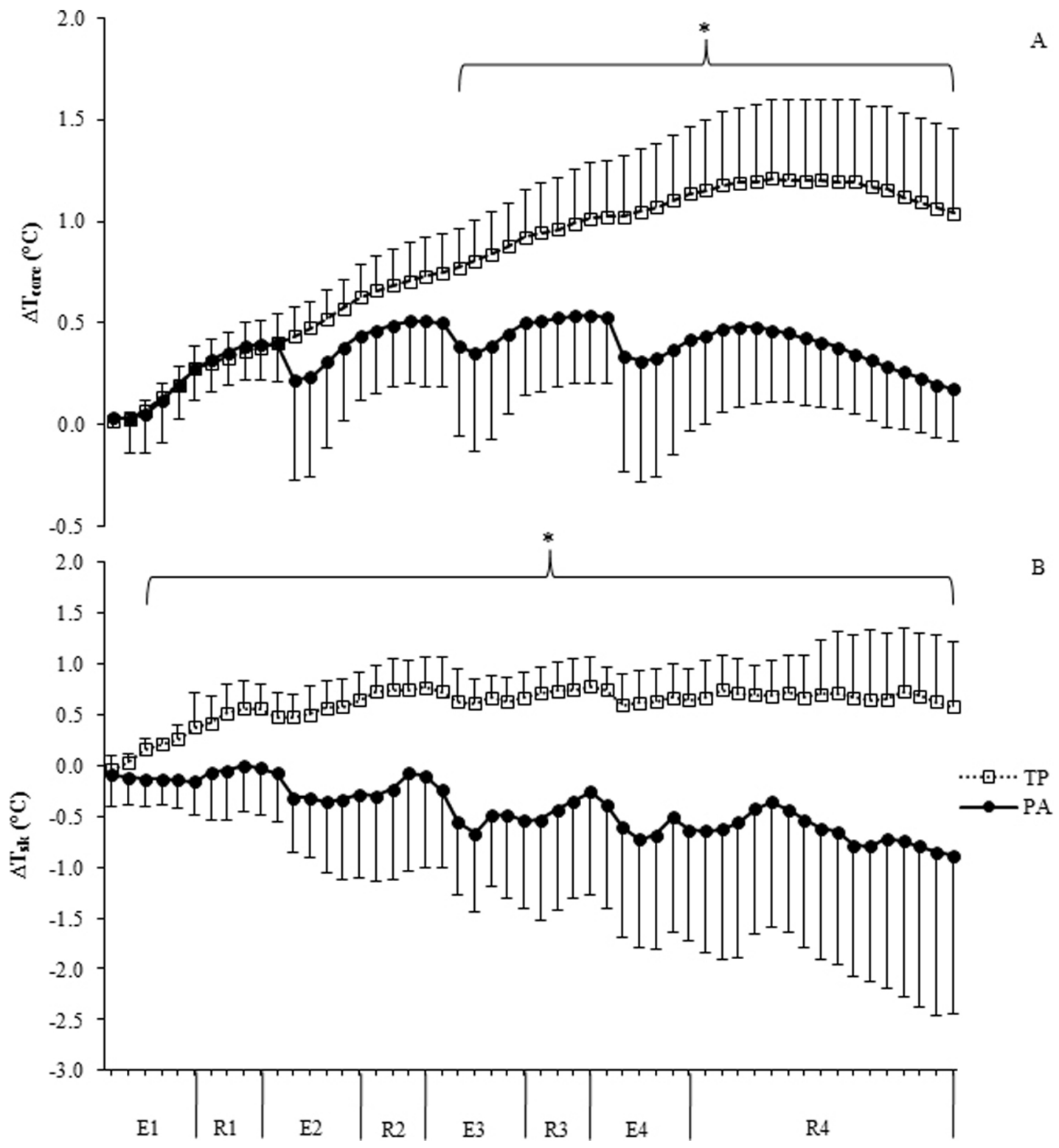

Figure 2 - Change in (A) core temperature $\left(T_{\text {core }}\right)$ and $(\mathrm{B})$ mean skin temperature $\left(T_{\mathrm{sk}}\right)$ from resting values during exercise $(\mathrm{E})$ and recovery $(\mathrm{R})$ for athletes with tetraplegia (TP) and athletes with paraplegia (PA) during each exercise block and recovery. $*$ Significantly different from PA $(P<.05)$.

$=1.43$ for exercise and recovery, respectively). For the PA group, $T_{\text {sk }}$ decreased during exercise, while athletes with TP experienced an increase in $T_{\mathrm{sk}}$ (Figure 2). Individual skin temperatures (Figure 3) were similar between groups at the start and end of exercise $(P>.05)$. During exercise, back skin temperature was the only site that demonstrated a difference between groups, with an increase from resting values in $\mathrm{TP}\left(0.9^{\circ} \mathrm{C} \pm 0.6^{\circ} \mathrm{C}\right)$ and a decrease in PA $\left(-0.4^{\circ} \mathrm{C} \pm 0.9^{\circ} \mathrm{C}\right.$, $P<.001$, ES $=1.65)$. During recovery, chest, back, forearm, and forehead skin temperature remained elevated from start-of-recovery values to a greater extent in TP than PA $(P<.05)$.

\section{Heat Storage}

Heat storage was greater in TP $(2.8 \pm 1.2 \mathrm{~J} / \mathrm{g})$ than PA $(1.0 \pm 1.0$ $\mathrm{J} / \mathrm{g}$ ) during exercise (Figure $4, P<.001, \mathrm{ES}=1.61$ ) and at the end of recovery $(3.4 \pm 1.4 \mathrm{~J} / \mathrm{g}$ and $-0.5 \pm 1.3 \mathrm{~J} / \mathrm{g}$ for $\mathrm{TP}$ and $\mathrm{PA}$, respectively, $P<.001$, ES = 3.08).

\section{Perceptual Measures}

During exercise, rating of perceived exertion was similar between groups $(P=.52, \mathrm{ES}=0.24)$, with an increase over time $(14 \pm 1$ and $16 \pm 2$ for the end of blocks 1 and 4 , respectively). Thermal sensation was similar between groups during exercise, $(4 \pm 1$ and $6 \pm 1$ at rest and end of block 4 , respectively, $P=.29, \mathrm{ES}=0.31)$ and recovery ( $6 \pm 1$ and $3 \pm 1$ at the start and end of recovery, respectively, $P=$ $.69, \mathrm{ES}=0.14)$.

\section{Fluid Balance}

Both TP and PA drank similar amounts during the ISP and recovery $(540 \pm 112$ and $469 \pm 233 \mathrm{~mL}$ for TP and PA, respectively, $P=.45$, $\mathrm{ES}=0.39)$. The change in body mass $(0.4 \pm 0.4$ and $0.1 \pm 0.3 \mathrm{~kg}$ for TP and PA, respectively, $P=.11, \mathrm{ES}=0.84$ ) and plasma volume changes were similar between groups $(4.0 \% \pm 13.7 \%$ and $4.3 \% \pm$ $9.5 \%$ for TP and PA, respectively, $P=.96, \mathrm{ES}=0.03$ ). 

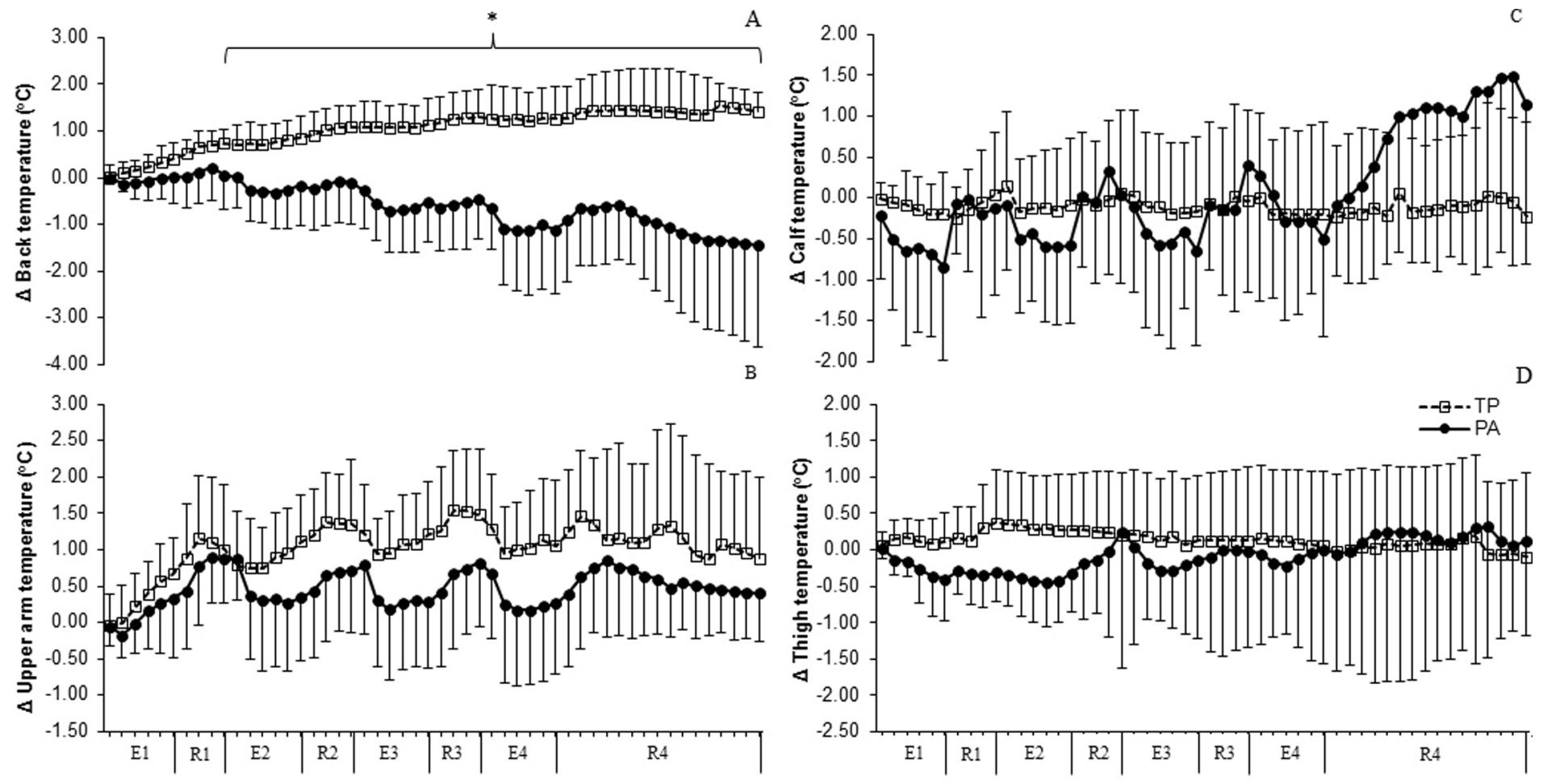

Figure 3 - Individual (A) back, (B) upper-arm, (C) calf, and (D) thigh skin temperatures for athletes with tetraplegia (TP) and athletes with paraplegia $(\mathrm{PA})$ during each exercise block $(\mathrm{E})$ and recovery $(\mathrm{R}) .{ }^{*}$ Significantly different from PA $(P<.05)$.

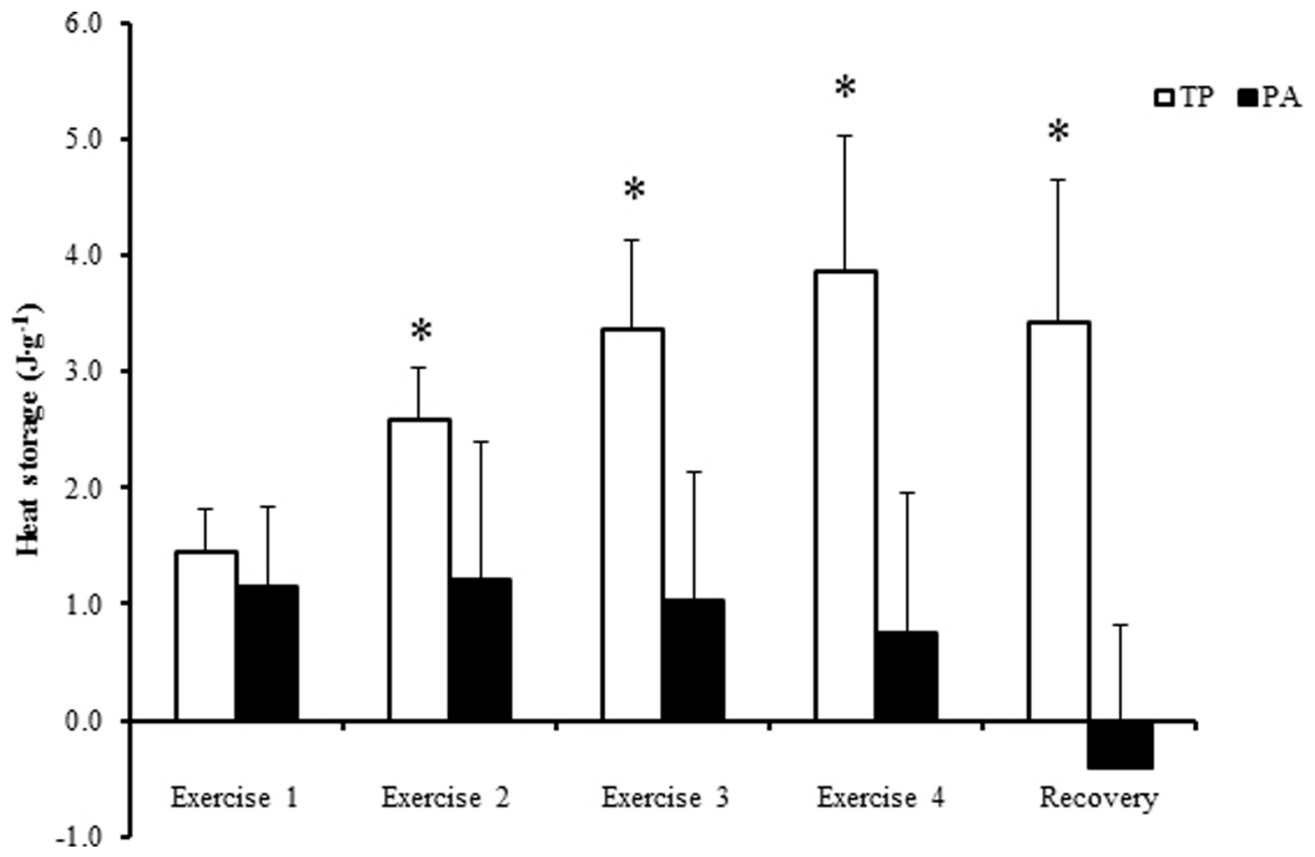

Figure 4 - Heat storage for athletes with tetraplegia $(\mathrm{TP})$ and athletes with paraplegia $(\mathrm{PA})$ during each exercise block and recovery. $*$ Significantly different from PA $(P<.05)$.

\section{Discussion}

The main findings indicate that despite external work being similar between groups, $T_{\text {core }}$ and heat storage increased at a greater magnitude in TP compared with PA during intermittent-sprint exercise in cool conditions. The greater increase in $T_{\text {core }}$ for TP signifies that thermoregulatory differences between the groups were predominantly due to a lower capacity for heat loss in TP than in PA. Even during postexercise recovery, $T_{\text {core }}$ and heat storage remained elevated in TP, signifying an inability to dissipate the heat produced during exercise, resulting in the retention of heat during recovery. 
Further increases in thermal strain in TP were not prevented by the active and passive recovery between the maximum-effort bouts, as $T_{\text {core }}$ and heat storage were found to continually increase throughout the protocol in this group. The $T_{\text {core }}$ responses for both groups are therefore comparable to those in previous studies during continuous wheelchair exercise, with increases of $0.2^{\circ} \mathrm{C}$ to $0.7^{\circ} \mathrm{C}^{6,22}$ and $0.9^{\circ} \mathrm{C}^{9}$ observed for PA and TP, respectively.

The $T_{\text {sk }}$ response of the 2 groups likely reflects the athletes' sweating capacity, being proportional to lesion level. For instance, the greater reduction in sweating capacity in TP resulted in an increase in $T_{\mathrm{sk}}$ during exercise. In PA, $T_{\mathrm{sk}}$ decreased during exercise, likely due to the larger body-surface area available for sweating and therefore greater evaporative cooling of the skin. It should be noted that although $T_{\mathrm{sk}}$ was not significantly different at the onset of exercise, a large ES demonstrates that PA may have had a substantially warmer starting $T_{\text {sk }}$ than TP. Nonetheless, $T_{\text {sk }}$ data should be interpreted with caution in individuals with a SCI, as it may mask regional skin-temperature responses. ${ }^{5}$

During exercise, differing responses in back skin temperature were apparent, increasing in TP and decreasing in PA, due to the majority of the upper-body skin of TP being insensate compared with sensate in PA. However, a similar finding was not found for chest skin temperature. Sweat rates vary with body region in ablebodied individuals, with a greater sweat rate apparent at the upper back than the chest. ${ }^{23}$ Therefore, at the chest, a lower evaporative cooling effect of sweat may have been apparent in PA, resulting in a chest skin temperature similar to that seen in TP. In both groups, upper-arm skin temperature demonstrated a decrease during exercise shown previously, yet more pronounced during continuous wheelchair propulsion. ${ }^{11}$ The decrease in upper-arm skin temperature is thought to be caused by the arm moving relative to the body in wheelchair propulsion, causing convective cooling to the upper arm. ${ }^{11}$

Neither group experienced a change in thigh skin temperature during exercise or recovery, likely due to the disrupted blood flow and vascular atrophy below the level of the lesion. ${ }^{3}$ Although small, there was a significant increase from rest in calf skin temperature over time, possibly due to the variable response of calf skin temperature in PA. ${ }^{10} \mathrm{~A}$ greater increase in calf skin temperature than the current study was previously observed during prolonged arm cranking, leading those authors to suggest that the lower body is a potential site for heat storage in PA. ${ }^{9,10}$ The degree of sweating and blood-flow redistribution in the lower limb may be dependent on the lowest intact part of the sympathetic chain, with the pathway for vasodilation in the lower limb located at or below T10. ${ }^{22}$ In individuals with lesions at T12, calf skin temperature has been shown to increase during exercise, with little or no change for individuals with lesions at T10-T11.22 However, in the current study, similar trends in calf skin temperature were apparent for individuals with lesions above $(n=5)$ and below T10 $(n=3)$ in the PA group. To fully understand the underlying mechanisms of vasomotor control of the lower body during upper-body exercise, further study is required.

More pronounced differences between skin-temperature sites may have been masked by the large interindividual variations in skin temperatures, a noticeable response in studies in the SCI population. ${ }^{24,25}$ These variations may have been heightened by the large range of lesion levels in PA (T4-S1), resulting in differences in sympathetic and somatosensory pathways, in arrangements of sympathetic outflow and the type and degree of reinnervation. ${ }^{3,10}$

From a perceptual perspective, even though the TP group was exercising at a greater $T_{\text {core }}$ than PA, similar thermal-sensation scores throughout exercise indicate that they did not perceive themselves to be warmer. This may be related to training status, with potentially a greater $T_{\text {core }}$ being better tolerated by the highly trained. Although not significant, a large ES in training hours $(E S=0.73)$ signifies that the TP participants in the current study were more highly trained and hence may have a better tolerance of greater $T_{\text {core values. Due }}$ to the smaller surface area of sensate skin in TP than in PA, it is also possible that TP may not perceive the increase in body temperature as effectively. ${ }^{26}$ During higher-intensity exercise and in warmer ambient conditions, this may be of more concern, especially as these athletes could potentially override perceived signs of thermal strain, putting themselves at risk for heat illness. ${ }^{26}$

The training status of the athletes with TP may have led to a greater development of their remaining musculature. ${ }^{27}$ Potentially, this may have enabled them to produce power outputs and external work similar to those with PA. The larger total resistance of the ergometerwheelchair system for TP was, however, likely caused by the differences in the mass of the wheelchairs used in wheelchair basketball and rugby, with heavier wheelchairs used in the latter ( 11-13 vs $15-19 \mathrm{~kg}$ ). The lower mean and peak HR in TP, due to the reduced sympathetic innervation of the heart, is consistent with previous studies. ${ }^{28}$ Although there was no significant difference in $\mathrm{VO}_{2 \text { peak }}$, a large ES signifies a meaningful difference between the groups, with previous research indicating an inverse relationship between lesion level and $\mathrm{VO}_{2 \text { peak. }}{ }^{28}$ The extent to which the athletes' aerobic fitness would have affected the results is unclear, yet future work matching the groups for training status may accentuate the differences in thermoregulatory responses due to the level of spinal lesion.

\section{Practical Applications}

Although neither group was under considerable thermal strain, the current study highlights that athletes with TP experience a greater increase in $T_{\text {core }}$ for the same external work load of intermittentsprint exercise than those with PA. Even though the protocol had greater ecological validity than previous studies due to the intermittent nature and use of wheelchair propulsion, the ISP may not have been wholly reflective of a wheelchair basketball or rugby match. Total distances covered were considerably shorter $(2316 \mathrm{~m})$ than the activity profiles of wheelchair rugby players during a match (4540 m). ${ }^{29}$ If the ISP were of a magnitude similar to that of match play, that is, greater metabolic work, the athletes may have experienced a greater thermal response, especially those with TP. Practically, support staff should closely monitor athletes with TP for signs of heat stress during wheelchair court sports and, if possible, apply appropriate cooling before, during, or after play.

A limitation of the study may be the inclusion of 4 individuals with an incomplete SCI (1 TP and 3 PA) in the mean group values. The degree of autonomic dysfunction may depend on the completeness of the injury, ${ }^{28}$ with incomplete lesions resulting in a greater amount of sensory information regarding one's thermal state and a greater capacity to sweat. ${ }^{26}$ Nevertheless, their inclusion was justified, as their $T_{\text {core }}$ and $T_{\text {sk }}$ responses were within $1 \mathrm{SD}$ of the mean response of each group.

\section{Conclusion}

Similarly to continuous arm-cranking and wheelchair exercise, athletes with TP have a greater inability to dissipate heat than those with PA during intermittent-sprint exercise in cool conditions. Despite the 2 groups' producing similar amounts of external work, the TP group had a marked increase in $T_{\text {core }}$ during exercise 
and recovery, signifying that differences between the groups were predominantly due to differences in heat loss. Neither group was under high levels of thermal strain, yet the current study highlights the heightened thermal response of athletes with TP to intermittent wheelchair exercise, with caution that a greater $T_{\text {core }}$ response may be apparent during actual game play. Support staff should be aware of the greater thermal impairment experienced by those with TP in wheelchair court sports, monitor them for signs of heat stress, and, if possible, apply appropriate cooling before, during, or after play.

\section{Acknowledgments}

The authors would like to thank GBWR and the Great Britain Wheelchair Basketball Association for their support and participation in the current study. Also we thank Jessica Lavin (formerly School of Sport, Exercise and Health Sciences, Loughborough University) and Dr John Lenton (Peter Harrison Centre for Disability Sport, School of Sport, Exercise and Health Sciences, Loughborough University) for their assistance during data collection, Craig Boyd (Faculty of Health and Wellbeing, Sheffield Hallam University) for the loan of equipment, Prof George Havenith (Design School, Loughborough University) for his guidance on the study, and Dr Steve Faulkner for proofreading the manuscript (School of Sport, Exercise and Health Sciences, Loughborough University).

\section{References}

1. Freund PR, Brengelmann GL, Rowell LB, Halar E. Attenuated skin blood flow response to hyperthermia in paraplegic men. J Appl Physiol. 1984;56(4):1104-1109. PubMed doi:10.1063/1.334081

2. Rawson RO, Hardy JD. Sweat inhibition by cutaneous cooling in normal sympathectomized and paraplegic man. J Appl Physiol. 1967;22(2):287-291. PubMed

3. Hopman MT. Circulatory responses during arm exercise in individuals with paraplegia. Int J Sports Med. 1994;15(3):126-131. PubMed doi:10.1055/s-2007-1021033

4. Normell LA. Distribution of impaired cutaneous vasomotor and sudomotor function in paraplegic man. Scand J Clin Lab Invest Suppl. 1974;138:25-41. PubMed

5. Price MJ. Thermoregulation during exercise in individuals with spinal cord injuries. Sports Med. 2006;36(10):863-879. PubMed doi:10.2165/00007256-200636100-00005

6. Dawson B, Bridle J, Lockwood R. Thermoregulation of paraplegic and able bodied men during prolonged exercise in hot and cool climates. Paraplegia. 1994;32(12):860-870. PubMed doi:10.1038/sc.1994.132

7. Price MJ, Campbell IG. Effects of spinal cord lesion level upon thermoregulation during exercise in the heat. Med Sci Sports Exerc. 2003;35(7):1100-1107. PubMed doi:10.1249/01. MSS.0000074655.76321.D7

8. Guttmann L, Silver J, Wyndham CH. Thermoregulation in spinal man. J Physiol. 1958;142(3):406-419. PubMed doi:10.1113/jphysiol.1958. sp006026

9. Price MJ, Campbell IG. Thermoregulatory responses of spinal cord injured and able-bodied athletes to prolonged upper body exercise and recovery. Spinal Cord. 1999;37(11):772-779. PubMed doi:10.1038/ sj.sc. 3100907

10. Price MJ, Campbell IG. Thermoregulatory responses of paraplegic and able-bodied athletes at rest and during prolonged upper body exercise and passive recovery. Eur J Appl Physiol Occup Physiol. 1997;76(6):552-560. PubMed doi:10.1007/s004210050289
11. Price MJ, Campbell I. Thermoregulatory and physiological responses of wheelchair athletes to prolonged arm crank and wheelchair exercise. Int J Sports Med. 1999;20(7):457-463. PubMed doi:10.1055/s-1999-8831

12. Kirshblum SC, Burns SP, Biering-Sorensen F, et al. International standards for neurological classification of spinal cord injury (revised 2011). J Spinal Cord Med. 2011;34(6):535-546. PubMed doi:10.117 9/204577211X13207446293695

13. Ramanathan NL. A new weighting system for mean surface temperature of the human body. J Appl Physiol. 1964;19:531-533. PubMed

14. Havenith G, Inoue Y, Luttikholt V, Kenney WL. Age predicts cardiovascular, but not thermoregulatory, responses to humid heat stress. Eur J Appl Physiol Occup Physiol. 1995;70(1):88-96. PubMed doi:10.1007/ BF00601814

15. Lenton JP, Fowler N, van der Woude L, Goosey-Tolfrey VL. Efficiency of wheelchair propulsion and effects of strategy. Int J Sports Med. 2008;29(5):384-389. PubMed doi:10.1055/s-2007-965569

16. Theisen D, Francaux M, Fayt A, Sturbois X. A new procedure to determine external power output during handrim wheelchair propulsion on a roller ergometer: a reliability study. Int J Sports Med. 1996;17(8):564-571. PubMed doi:10.1055/s-2007-972896

17. Leicht CA, Bishop NC, Goosey-Tolfrey VL. Mucosal immune responses during court training in elite tetraplegic athletes. Spinal Cord. 2012;50(10):760-765. PubMed doi:10.1038/sc.2012.47

18. Borg G. Perceived exertion as an indicator of somatic stress. Scand J Rehabil Med. 1970;2(2):92-98. PubMed

19. Toner MM, Drolet LL, Pandolf KB. Perceptual and physiological responses during exercise in cool and cold water. Percept Mot Skills. 1986;62(1):211-220. PubMed doi:10.2466/pms.1986.62.1.211

20. Dill DB, Costill DL. Calculation of percentage changes in volumes of blood, plasma, and red cells in dehydration. J Appl Physiol. 1974;37(2):247-248. PubMed

21. Cohen J. Statistical Power Analysis for the Behavioral Sciences. 2nd ed. Hillsdale, NJ: Lawrence Earlbaum; 1988.

22. Gass GC, Camp EM, Nadel ER, Gwinn TH, Engel P. Rectal and rectal vs. esophageal temperatures in paraplegic men during prolonged exercise. J Appl Physiol. 1988;64(6):2265-2271. PubMed

23. Smith CJ, Havenith G. Body mapping of sweating patterns in male athletes in mild exercise-induced hyperthermia. Eur J Appl Physiol. 2011;111(7):1391-1404. PubMed doi:10.1007/s00421-010-1744-8

24. Gass EM, Gass GC, Gwinn TH. Sweat rate and rectal and skin temperatures in tetraplegic men during exercise. Sports Med Train Rehabil. 1992;3(4):243-249. doi:10.1080/15438629209511951

25. Price MJ, Campbell IG. Thermoregulatory responses during prolonged upper-body exercise in cool and warm conditions. J Sports Sci. 2002;20(7):519-527. PubMed doi:10.1080/026404102760000026

26. Webborn N, Price MJ, Castle P, Goosey-Tolfrey V. Cooling strategies improve intermittent sprint performance in the heat of athletes with tetraplegia. Br J Sports Med. 2010;44(6):455-460. PubMed doi:10.1136/bjsm.2007.043687

27. Abel T, Platen P, Rojas Vega S, Schneider S, Struder HK. Energy expenditure in ball games for wheelchair users. Spinal Cord. 2008;46(12):785-790. PubMed doi:10.1038/sc.2008.54

28. Theisen D. Cardiovascular determinants of exercise capacity in the Paralympic athlete with spinal cord injury. Exp Physiol. 2012;97(3):319-324. PubMed doi:10.1113/expphysiol.2011.063016

29. Sarro KJ, Misuta MS, Burkett B, Malone LA, Barros RML. Tracking of wheelchair rugby players in the 2008 demolition derby final. J Sports Sci. 2010;28(2):193-200. PubMed doi:10.1080/02640410903428541 